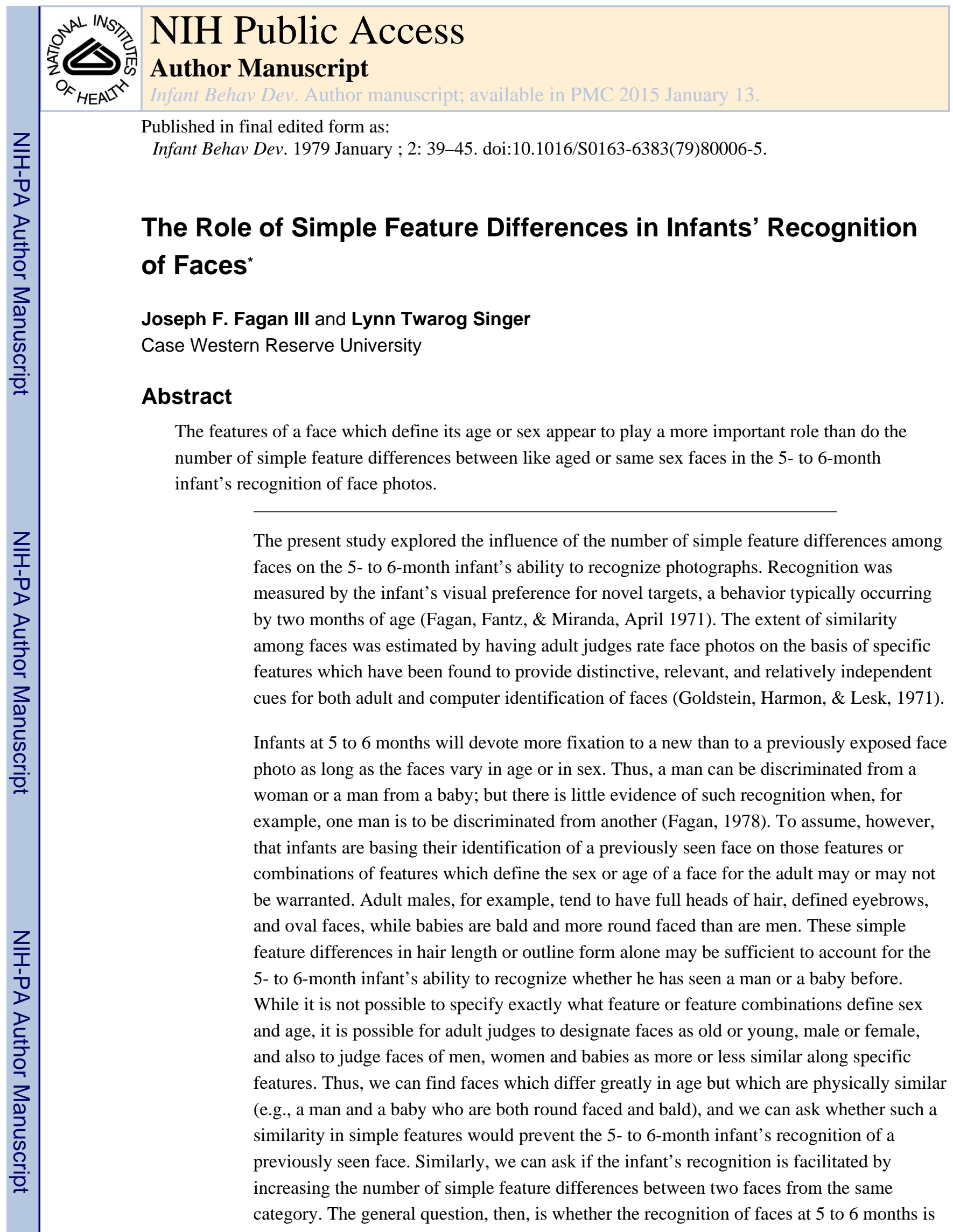

\footnotetext{
* The present study was supported by Multiple Research Project Grant HD-11089 and Career Development Grant 1K04 HD-70144 from the National Institute of Child Health and Human Development and by a grant from the National Foundation. Joseph F. Fagan's address is Department of Psychology, Case Western Reserve University, Cleveland, Ohio 44106.
} 
primarily accomplished by an analysis of the particular information defining sex or age or by a reliance on the more obvious physical differences along certain face features which covary with age or sex. More specifically, the present study asked some 5- to 6-month-old infants to recognize which of two faces they had seen before when the two faces were different in age or in sex but were judged by adults as having highly similar physical features. Other infants were asked to identify which face was new when the two faces were alike in sex and in age but were judged by adults as being quite disparate when scaled along specific features.

\section{METHOD Subjects}

The sample consisted of 120 Caucasian infants (64 males, 56 females). The mean age of the group was 22.2 weeks, SD 0.5 weeks, range 20.6-23.4 weeks.

\section{Apparatus and Materials}

All subjects were tested at home by means of a portable apparatus consisting of a testing chamber containing a pivoting stimulus-presentation "stage." Removable $17.5 \times 17.5 \mathrm{~cm}$ stimulus plaques were attached to the stage. When in place, the two plaques were $30.5 \mathrm{~cm}$ apart from center to center and approximately $30.0 \mathrm{~cm}$ from the infant's eyes. Through a 64 $\mathrm{cm}$ peephole in the center of the stage, the observer could see corneal reflections of stimulus targets. Length of superimposition of the left or right reflection over the pupil of either eye was recorded. Inter-observer reliability in the measurement of differential fixation employing the same criteria as the present study has been found to be uniformly high by a number of investigators. A review is provided in Fagan (1973).

The achromatic face photos employed in the present study are illustrated in Fig. 1. Each face measured approximately $13.8 \mathrm{~cm}$ from crown to chin to obtain faces of different age or sex which were approximately equal in size, and each was then mounted on a $15 \times 15 \mathrm{~cm}$ square of white posterboard. The three pairings pictured in the left column, the baby and the bald man, the white man and white woman (in actuality, brother and sister), and the black man and black woman, had been rated by adult judges as having relatively few feature differences between the pair members. The two members in each of the three remaining pairings, the two men, the two white women, and the black woman and white woman pictured in the right column, although the same sex, were judged by adults as quite dissimilar. Specifically, each photo had been scaled by adult judges along 17 of the 21 face features listed by Harmon (1973). These features included the coverage, length, texture, and shade of the hair; the weight and separation of eyebrows; the opening, separation, and shade of the eyes; the protrusion of the ears; the fullness of the cheeks; the length, tip-direction, and profile-form of the nose; and the upper lip thickness, lower lip thickness, and width of the mouth. The four remaining features (forehead profile shape, ear length, lip overlap, and chin profile) could only be judged from profile poses and, hence, were omitted from consideration. The judges (11 males and 13 females) were students, staff, or faculty members in the psychology department. Faces B, $\mathbf{M}_{1}, \mathrm{M}_{2}$ were rated by nine judges; faces $\mathrm{M}_{3}, \mathrm{~W}_{1}, \mathrm{~W}_{2}, \mathrm{~W} 3$ by 10 judges; and faces $\mathrm{M}_{4}, \mathrm{~W}_{4}, \mathrm{~W}_{5}$ by 10 judges. Each face was rated 
along a five-point scale on each feature with the exception of eyebrow separation which was rated along a three-point scale. The instructions were to rate each face on an absolute basis, i.e., not comparing one face with another insofar as possible. A face was rated for all 17 features before the next photo (order of photos randomly determined) was rated. Interjudge reliability coefficients computed for each feature were uniformly high with a median of .95 . The extent of physical dissimilarity between each of the six pairings of photos pictured in Fig. 1 was computed by counting the number of features on which members of a pair had received reliably different ratings (correlated $t$-tests, with $p<.02$ ). Thus, the baby and the bald man were seen to differ on five of the 17 features, while the bald man and the darkhaired man $\left(\mathrm{M}_{2}\right)$ differed on ten out of 17 features (including three which differentiated the baby from the bald man). The photos of a brother and sister $\left(\mathrm{M}_{3}\right.$ and $\left.\mathrm{W}_{1}\right)$ were seen as highly similar with only two distinguishing features. The pairing of the two white women $\left(\mathrm{W}_{2}\right.$ and $\mathrm{W}_{3}$ ) proved to have seven reliable feature differences (including one of the two which varied between the brother and the sister). The black man and black woman ( $\mathrm{M}_{4}$ and $\left.\mathrm{W}_{4}\right)$ differed on four features; the black woman and white woman $\left(\mathrm{W}_{4}\right.$ and $\left.\mathrm{W}_{5}\right)$ varied on six features, quite apart from the brightness differences due to skin color. In summary, adult judges provided reliable ratings of face photos along specific features. More important for our present purposes is the fact that pairings of faces disparate in age or in sex (baby-man; man-woman) were seen as having fewer intra-pair feature differences than were pairings of a man with a man or woman with a woman.

Five- to 6-month infants in a previous study (Fagan, 1977) did not differentiate the man labeled $\mathrm{M}_{3}$ in Fig. 1 from a highly similar man with a full smile, a smile such as the one worn by $\mathrm{W}_{1}$ and $\mathrm{W}_{2}$. Nevertheless, an effort was made in the present study to control for differences in expresssion between faces (e.g., full smile with teeth exposed vs. slight smile with mouth closed) by ensuring that similar expression differences were present for the same-sex pairing of the two white women as well as for the different-sex pairing of the brother and sister.

\section{Procedure}

Separate groups of infants were asked to discriminate, on a recognition task, among the six pairs of faces shown in Fig. 1. An additional sample of 16 infants were tested on a pairing of the baby with $\mathrm{M}_{2}$. The specific procedure was to expose each infant to one target for two 30sec study periods (e.g., the baby's face would be shown once on the left and once on the right of the stage) and then to give a 20 -sec recognition test by pairing the previously exposed target with a novel target for two 10-sec periods, reversing left-right positions from one period to the next (e.g., baby-bald man, bald man-baby). The two photos making up a particular recognition task served equally often as novel or familiar. Half the infants in each group were tested by one observer and half by another.

\section{RESULTS AND DISCUSSION}

The most important aspects of the data, the amount of fixation elicited during the study period and the percentage of total fixation paid to the novel relative to the familiar target during recognition testing, are listed in Fig. 1 under the heading of Fix, and \% N, respectively. Preliminary analysis indicated that fixation times and responsiveness to novelty 
were not influenced by the sex of the subject and, further, that subjects in each group tested by the different observers yielded highly comparable scores. Hence, results from both sexes and from each observer were pooled in presenting the summary data listed in Fig. 1. As is evident, amounts of study time during the 60 -sec study periods were comparable for the six groups ranging from 36.7 to $43.3 \mathrm{sec}$. The 16 infants tested on baby vs. $\mathrm{M}_{2}$ fixated the target presented during study for an average of 36.1 seconds, SD 9.0. The 120 subjects averaged $14.4 \mathrm{sec}$ of fixation, SD 4.0, during the 20 -sec recognition testing period. The percentages of total fixation paid to the novel target on recognition testing varied over groups. The $t$ values in Fig. 1 represent the departure of the mean $\% \mathrm{~N}$ score from a chance value of $50 \%$. Faces disparate in age or in sex but judged similar along many features (the bald man and the baby, the brother and sister, the black man paired with the black woman) were easily discriminated, as demonstrated by the high and reliable preferences for novelty of $65.3 \%$, $63.2 \%$, and $61.1 \%$, respectively. Infants gave no evidence, however, of having recognized which of two highly dissimilar men or women had previously been seen, with low and unreliable preferences of $54.3 \%, 50.2 \%$, and $52.0 \%$, respectively, paid to the novel members of the pairs. Moreover, the differences between the mean $\% \mathrm{~N}$ score of $63.5 \%$, SD 16.9, for the 58 infants tested on between sex or age discriminations and the mean of $52.4 \%$, SD 16.7, for the 46 infants given within sex or age distinctions was highly reliable $(t(102)=3.9, p<$. 001). The additional 16 infants tested on a pairing of the baby with the dark-haired man $\left(\mathrm{M}_{2}\right)$ are of particular interest. Adults had rated $\mathrm{B}$ and $\mathrm{M}_{2}$ as differing on 11 out of 17 features, the greatest number of simple feature differences of any pairing. Although the baby and $\mathrm{M}_{2}$ were different in age as well as being highly dissimilar physically, the resulting novelty preference of $64.3 \%$, SD $16.1(t(15)=3.5, p<.01)$, was no greater than the $65.3 \%$ elicited by the unliked aged but physically similar pairing of the baby and the bald man.

In effect, whatever the features constituting the differences between a baby and a man or between a man and a woman, these attributes seem to be sufficient for the 5- to 6-month infant's recognition with no added advantage for recognition when simple physical differences are maximized. On the other hand, it would appear that extensive physical differences without accompanying differences in age or in sex are not sufficient for recognition, as least under the stimulus and temporal test conditions employed in the present study. In a similar vein, Dirks and Gibson (1977) found that 5-month infants could distinguish between chromatic photos of a man and a woman following exposure to the actual man or woman but evidenced no such distinctions when two men or two women were to be differentiated. They noted that same-sex faces were also alike in hair style and in hair color and such obvious physical similarities may have contributed to the infants' failure to discriminate among men or among women. The present results indicate that it was the similarity in sex per se, rather than the similarities in simple features such as hair length or color, which prevented recognition on the part of the infants in the Dirks and Gibson 1977 study.

Evidence from studies of auditory discrimination (Eimas, 1977) and color perception (Bornstein, 1977) have shown that category membership rather than degree of physical similarity is the more important factor in aiding infant recognition. Although the objective criteria for defining the age or sex of a face cannot be specified as can those for phonemes or 
hue classification, the present results indicate that infants at 5 months are more apt to base their memory for face photos on those features or feature combinations (whatever they may be) that define sex or age rather than on the number of simple feature differences among faces. However, by 7 months, the number of simple feature differences among faces does aid the infant in differentiating between two male faces (Fagan, 1976). Hence, it would appear that particular features or feature combinations defining the sex or age of a face are responded to or relied on for recognition earlier in development than are differences along more easily specified face features. The specification of the exact characteristics which define the age or sex of a face for the infant remain interesting conceptual and empirical questions for further study.

\section{References}

Bornstein, MH. Chromatic vision in infancy. In: Reese, HW.; Lipsitt, LP., editors. Advances in child development and behavior. Vol. 12. New York: Academic Press; 1977.

Dirks J, Gibson EJ. Infants' perception of similarity between live people and their photographs. Child Development. 1977; 48:124-130. [PubMed: 844350]

Eimas, PD. Speech perception in early infancy. In: Cohen, L.; Salapatek, P., editors. Infant perception: From sensation to cognition, Vol. 2. Perception of space, speech, and sound. New York: Academic Press; 1977.

Fagan JF. Infants' delayed recognition memory and forgetting. Journal of Experimental Child Psychology. 1973; 16:424-450. [PubMed: 4771431]

Fagan JF. Infants' recognition of invariant features of faces. Child Development. 1976; 47:627-638.

Fagan JF. Infant recognition memory: Studies in forgetting. Child Development. 1977; 48:68-78. [PubMed: 844363]

Fagan, JF. The origins of facial pattern recognition. In: Bornstein, MH.; Kessen, W., editors. Psychological development in infancy: Image to intention. Hillsdale, N. J: Lawrence Erlbaum Associates; 1978.

Fagan, JF.; Fantz, RL.; Miranda, SB. Infants' attention to novel stimuli as a function of postnatal and conceptual age. Paper, presented at the meeting of the Society for Research in Child Development; Minneapolis. April 1971;

Goldstein AJ, Harmon LD, Lesk AB. Identification of human faces. Proceedings of the IEEE. 1971; 59:748-760.

Harmon LD. The recognition of faces. Scientific American. 1973; 229:70-84. 

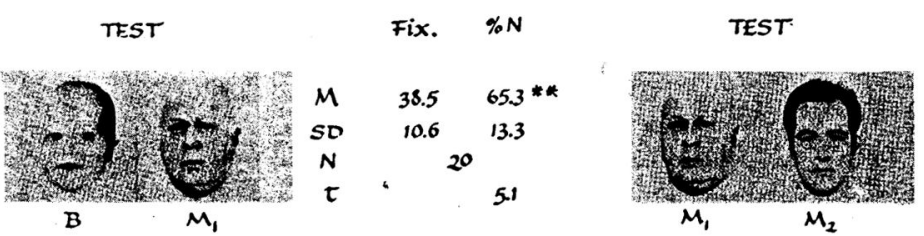

Fix. $\% N$
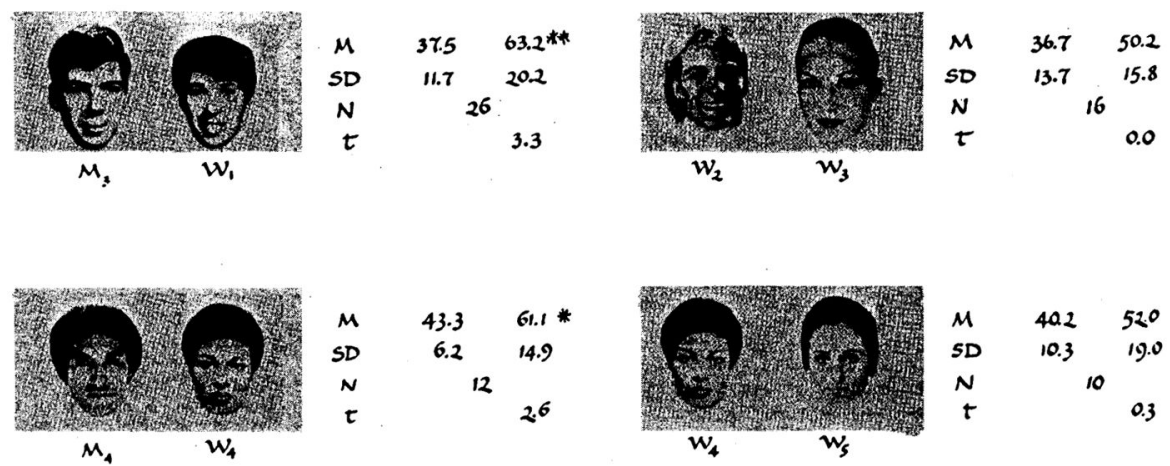

$* * p<.001$

Fig. 1.

Infant Behav Dev. Author manuscript; available in PMC 2015 January 13. 